
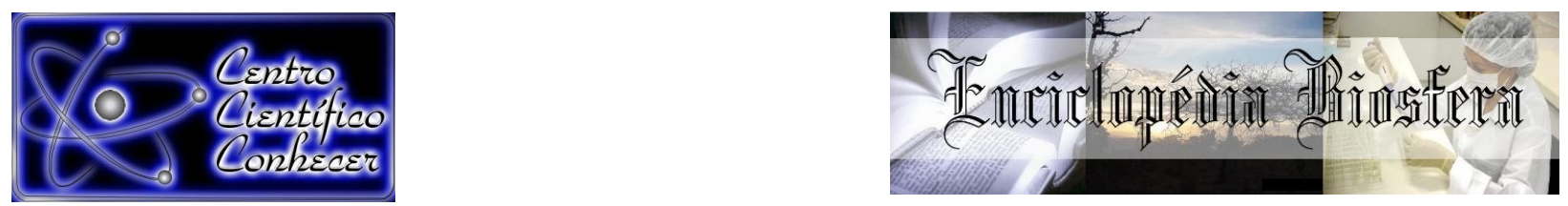

\title{
CONHECIMENTO, ATITUDE E PRÁTICA DO EXAME PAPANICOLAOU POR MULHERES DO NORTE DO ESPÍRITO SANTO
}

\author{
Luara Xavier Sena ${ }^{1}$, Nara de Almeida Souza ${ }^{1}$, Débora Barreto Teresa Gradella ${ }^{2}$ \\ ${ }^{1}$ Farmacêutica pela Universidade Federal do Espirito Santo, São Mateus, ES - Brasil \\ ${ }^{2}$ Professora Associada do Departamento de Ciências da Saúde da Universidade Federal do \\ Espirito Santo (debora.gradella@ufes.br), São Mateus, ES - Brasil
}

\section{Recebido em: 06/04/2018 - Aprovado em: 10/06/2018 - Publicado em: 20/06/2018 DOI: 10.18677/EnciBio_2018A98}

\begin{abstract}
RESUMO
O câncer cervical é uma das mais preocupantes neoplasias que acometem as mulheres e possui como principal estratégia de rastreamento o exame Papanicolaou. Assim, este estudo teve por objetivo avaliar o conhecimento, atitude e prática do exame preventivo por mulheres do norte do Espírito Santo, associando tais parâmetros com variáveis socioeconômicas. Tratou-se de um estudo descritivo transversal com abordagem quantitativa, no qual foram aplicados formulários em 360 mulheres atendidas em duas Unidades Básicas de Saúde do município de São Mateus-ES. Para análise de associação foi aplicado o teste qui-quadrado, com nível de significância de 5\%. Constatou-se que o conhecimento e a prática do exame citopatológico foram adequados em $78,06 \%$ e $95,00 \%$ das mulheres, respectivamente, embora apenas $61,94 \%$ das entrevistadas demonstraram atitude adequada. O sentimento de vergonha e a dificuldade para marcação de consulta foram relatados como barreiras para a realização do exame. Este cenário exige o aperfeiçoamento do método de trabalho, sendo necessárias melhorias no atendimento das ações de saúde da mulher a fim de estabelecer uma relação de confiança entre a paciente e o profissional de saúde.
\end{abstract}

PALAVRAS-CHAVE: câncer cervical; Papanicolaou; saúde da mulher

\section{KNOWLEDGE, ATTITUDE AND PRACTICE RELATED TO PAP TEST BY NORTHERN WOMEN OF ESPIRITO SANTO}

\begin{abstract}
The cervical cancer is one of the most worrying cancers affecting women and it has as its main screening strategy the Pap test. This study aimed to evaluate the knowledge, attitude and practice of the Pap test by northern women of Espírito Santo, linking these parameters to socioeconomic variables. This was a quantitative, descriptive and cross-sectional study in which a form was used to interview 360 women seen at two Basic Health Units in São Mateus-ES. To association analysis the chi-square test was used at a $5 \%$ significance level. It was found that the knowledge and practice of the Pap test were adequate in $78.06 \%$ and $95.00 \%$ of women, respectively, although only $61.94 \%$ of interviewed demonstrated adequate attitude. The feeling of shame and the difficult to mark consultation were established as barriers to the exam. This scenario requires the improvement of working methods, requiring improvements in women's health activities in order to establish a relationship of trust between the patient and the healthcare professional.
\end{abstract}

KEYWORDS: cervical cancer; Papanicolaou; women's health 


\section{INTRODUÇÃO}

O câncer do colo do útero (CCU) é caracterizado pela replicação desordenada do epitélio que reveste o útero e apresenta-se como um problema de saúde pública. É um dos tumores mais incidentes entre as mulheres e também uma importante causa de mortes por neoplasia na população feminina mundial. No Brasil, o câncer cervical ocupa a terceira posição entre os cânceres de maior incidência, instalando-se como uma das mais preocupantes neoplasias que acometem as mulheres, principalmente em regiões menos desenvolvidas. Isso pode estar relacionado com as dificuldades no acesso e a precariedade dos serviços de saúde que visam o diagnóstico e tratamento da doença (OPAS 2013; INCA, 2017a; IARC, 2017).

A etiologia do câncer cervical está comumente relacionada a uma prolongada infecção pelo Papilomavírus Humano (HPV), com grande probabilidade de desenvolvimento do câncer. Atualmente já são descritos mais de 150 tipos virais de HPV, dos quais 13 tipos são de alto risco oncogênico por provocarem infecções persistentes e estarem associados a lesões precursoras. Os HPV 16 e 18 são os tipos mais identificados e representam $70 \%$ de todos os tipos virais envolvidos no cancro invasivo (INCA, 2017c).

Embora o HPV seja um fator precursor na gênese do câncer do colo de útero, existem cofatores que podem potencializar sua ação e contribuir para o desenvolvimento da neoplasia cervical. Entre os fatores de risco mais importantes estão: o uso prolongando de contraceptivos hormonais; o início precoce da atividade sexual; a multiplicidade de parceiros; a multiparidade; o tabagismo; uma infecção pré-existente com o HIV, Chlamydia trachomatis e Herpes vírus; a imunossupressão; os distúrbios nutricionais; entre outros (MUÑOZ et al., 2006).

Apesar da alta incidência e de vários fatores de risco, o câncer cérvicouterino apresenta grande potencial de prevenção, visto que o desenvolvimento passa por vários estágios de lesões intra-epiteliais pré-cancerosas antes de alcançar à forma invasiva. A lenta evolução associada com a facilidade de diagnóstico possibilita a detecção da doença ainda nos estágios iniciais, um período em que há alto índice de cura, desde que a mulher realize o exame Papanicolaou regularmente. Este exame consiste na principal estratégia para detectar lesões precursoras e fazer o diagnóstico do câncer de colo uterino, além de poder avaliar outras condições como infecção e inflamação do trato genital feminino (BRASIL, 2013; INCA, 2017b).

Pesquisas apontam que uma boa cobertura do exame citopatológico demonstra ser eficaz na redução da mortalidade e incidência da doença em vários países. A qualidade do programa de rastreio depende da organização do serviço prestado, equidade de acesso, do envolvimento da população-alvo, bem como da periodicidade de realização do exame. A alta cobertura do teste Papanicolaou é de extrema importância nos indivíduos mais susceptíveis, sendo esta a atividade da atenção primária mais importante para combater as crescentes incidências e taxas de mortalidade do câncer de colo de útero (ARBYN et al., 2009).

Diante dos danos ocasionados pelo câncer cervical na vida de milhões de mulheres e da consolidação como um problema de saúde pública, tornou-se relevante a realização desse estudo, que teve por objetivo avaliar o conhecimento, atitude e prática das mulheres em relação ao exame Papanicolaou. Para isto, considerou-se necessária a análise de variáveis socioeconômicas, além da identificação de fatores associados à baixa adesão ao exame. 


\section{MATERIAL E MÉTODOS}

Tratou-se de um estudo descritivo transversal com abordagem quantitativa associado ao inquérito Conhecimento, Atitude e Prática (CAP), sendo realizado com mulheres atendidas em duas Unidades Básicas de Saúde do município de São Mateus-ES. O público-alvo da pesquisa foi constituído por mulheres cadastradas nas unidades, na faixa etária dos 25 aos 64 anos e em condições de compreender e responder as questões da pesquisa. Justifica-se a escolha da faixa etária em razão das orientações do Ministério da Saúde, que indica este grupo etário como preferencial na prevenção do câncer de colo uterino (BRASIL, 2013).

O cálculo para amostra aleatória simples foi realizado com base no número estimado de mulheres residentes no município segundo Censo Demográfico 2010 (IBGE, 2010), considerando nível de confiança de $95 \%$, erro amostral de $5 \%$, poder estatístico de $80 \%$, efeito de delineamento de 1 , perfazendo um total final de 360 mulheres.

Foram considerados como critérios de inclusão: mulheres residentes no município, dentro da faixa etária estabelecida, que concordaram em fazer parte da pesquisa e se dispuseram a responder a entrevista por meio de um formulário estruturado, aplicado por acadêmicas do curso de Farmácia. Este instrumento consistiu em uma entrevista contendo informações pessoais (idade, escolaridade, renda, local de residência, estado civil e número de filhos) e questões que abordavam os conhecimentos, atitudes e práticas sobre o exame Papanicolaou. Para análise dos dados foi utilizado o método adaptado de Brenna e colaboradores (2001), que consiste nas seguintes definições:

a) conhecimento adequado: quando a mulher já tinha ouvido falar do exame e sabia que era para detectar o câncer do colo uterino;

b) conhecimento inadequado: quando a mulher nunca tinha ouvido falar do exame ou já tinha ouvido, mas não sabia que era para detectar o câncer cérvico-uterino;

c) atitude adequada: quando a mulher acreditava que fazer o exame periodicamente era necessário e o realizava para prevenir o câncer do colo do útero;

d) atitude inadequada: quando a mulher acreditava que fazer o exame era necessário, mas não o realizava para prevenção do câncer cervical ou quando acreditava ser pouco necessário, desnecessário ou não apresentava opinião sobre a questão;

e) prática adequada: quando a mulher realizou seu último exame no máximo há três anos;

d) prática inadequada: quando a mulher havia realizado o último exame há mais de três anos, realizava o exame raramente ou nunca havia feito.

Os dados foram lançados no Microsoft Excel for Windows ${ }^{\circledR}$, versão 2013, e analisados por meio do programa estatístico GraphPad InStat 3.1. Realizou-se análise descritiva com estudo de frequência e percentagem, e para associação foi aplicado o teste qui-quadrado, considerando significativo valor de $p<0,05$.

O projeto foi submetido ao Comitê de Ética de Pesquisa com Seres Humanos, de acordo com a Resolução 466/2012 do Conselho Nacional de Saúde, e aprovado sob o $n^{\circ}$ CAAE: 34443214.5.0000.5063, sendo que todas as mulheres participantes assinaram o termo de consentimento livre e esclarecido.

\section{RESULTADOS E DISCUSSÃO}

Participaram do estudo 360 mulheres com média de idade de 39,86 anos $(D P=10,34)$. Destas, a maioria $(67,78 \%)$ estava na faixa etária de 25 a 44 anos, possuía companheiro $(76,11 \%)$, tinha de um a três filhos $(73,33 \%)$, apresentava 
renda familiar de um a dois salários mínimos $(64,44 \%)$ e escolaridade abaixo de ensino médio incompleto (52,22\%), sendo 3,06\% analfabetas (Tabela 1 ).

Entre as entrevistadas, 88,89\% iniciaram a atividade sexual com mais de 15 anos de idade e $10,83 \%$ com idade igual ou inferior a 14 anos. A maior parte das mulheres $(54,17 \%)$ afirmava não utilizar preservativo nas relações sexuais e $5,56 \%$ já haviam sido infectadas pelo Papilomavírus Humano, desenvolvendo verrugas genitais ou até mesmo o câncer do colo do útero (Tabela 1).

Quando questionadas sobre a participação em atividades educativas sobre doenças sexualmente transmissíveis, HPV ou câncer de colo de útero, 178 mulheres $(49,44 \%)$ afirmaram terem participado pelo menos uma vez e 133 dessas $(74,72 \%)$ informaram que estas atividades foram realizadas em Unidades Básicas de Saúde.

De acordo com as características socioeconômicas, constatou-se a influência da idade das mulheres sobre o conhecimento e a prática do exame. $O$ número de filhos e o grau de escolaridade associaram-se com a atitude das mulheres. No que diz respeito a vida sexual das mulheres, observou-se que o início precoce das relações sexuais pode resultar na prática inadequada do exame preventivo. A renda familiar e infecção prévia por HPV não apresentaram nenhuma relação com os parâmetros estudados (Tabela 1).

TABELA 1. Conhecimento, atitude e prática das mulheres sobre o exame Papanicolaou de acordo com variáveis socioeconômicas. São Mateus, ES, Brasil, 2015. $(\mathrm{N}=360)$ 


\begin{tabular}{|c|c|c|c|c|c|c|c|c|c|c|}
\hline \multirow[t]{2}{*}{ Variável } & \multicolumn{4}{|c|}{$\begin{array}{c}\text { Conhecimento } \\
\text { adequado }\end{array}$} & \multicolumn{3}{|c|}{ Atitude adequada } & \multicolumn{3}{|c|}{ Prática adequada } \\
\hline & Total & $\mathrm{n}$ & $\%$ & $\mathrm{P}$ & $\mathrm{N}$ & $\%$ & $\mathrm{P}$ & $\mathrm{n}$ & $\%$ & $p$ \\
\hline \multicolumn{11}{|l|}{ Idade (anos) } \\
\hline $25-44$ & 244 & 181 & 74,18 & 0,015 & 159 & 65,16 & 0,088 & 227 & 93,03 & 0,026 \\
\hline $45-64$ & 116 & 100 & 86,21 & & 64 & 55,17 & & 115 & 99,14 & \\
\hline \multicolumn{11}{|l|}{ Escolaridade } \\
\hline Analfabeta & 11 & 7 & 63,64 & 0,647 & 5 & 45,45 & 0,003 & 10 & 90,91 & 0,204 \\
\hline Fundamental incompleto & 114 & 88 & 77,19 & & 60 & 52,63 & & 108 & 94,74 & \\
\hline Fundamental completo & 38 & 30 & 78,95 & & 19 & 50,00 & & 37 & 97,37 & \\
\hline Médio incompleto & 36 & 28 & 77,78 & & 20 & 55,56 & & 31 & 86,11 & \\
\hline Médio completo & 133 & 104 & 78,20 & & 96 & 72,18 & & 129 & 96,99 & \\
\hline Superior incompleto & 10 & 10 & 100,00 & & 9 & 90,00 & & 10 & 100,00 & \\
\hline Superior completo & 18 & 14 & 77,78 & & 14 & 77,78 & & 17 & 94,44 & \\
\hline \multicolumn{11}{|l|}{ Renda familiar* } \\
\hline Não possui & 23 & 18 & 78,26 & 0,196 & 17 & 73,91 & 0,097 & 22 & 95,65 & 0,519 \\
\hline Até 1 & 43 & 32 & 74,42 & & 22 & 51,16 & & 39 & 90,70 & \\
\hline 1 a 2 & 232 & 177 & 76,29 & & 140 & 60,34 & & 220 & 94,83 & \\
\hline 3 a 4 & 57 & 51 & 89,47 & & 39 & 68,42 & & 56 & 98,25 & \\
\hline$\geq 5$ & 5 & 3 & 60,00 & & 5 & 100,00 & & 5 & 100,00 & \\
\hline \multicolumn{11}{|l|}{ Companheiro } \\
\hline Não possui & 86 & 69 & 80,23 & 0,682 & 50 & 58,14 & 0,480 & 81 & 94,19 & 0,910 \\
\hline Possui & 274 & 212 & 77,37 & & 173 & 63,14 & & 261 & 95,26 & \\
\hline \multicolumn{11}{|l|}{$N^{0}$ de filhos } \\
\hline Não possui & 29 & 17 & 58,62 & 0,055 & 19 & 65,52 & 0,011 & 25 & 86,21 & 0,064 \\
\hline 1 a 3 & 264 & 213 & 80,68 & & 168 & 63,64 & & 254 & 96,21 & \\
\hline 4 a 5 & 50 & 38 & 76,00 & & 32 & 64,00 & & 48 & 96,00 & \\
\hline$>5$ & 17 & 13 & 76,47 & & 4 & 23,53 & & 15 & 88,24 & \\
\hline
\end{tabular}

Início da atividade

sexual

$\leq 14$ anos

15 a 18 anos

$>18$ anos

$\begin{array}{ccc}39 & 27 & 69,23 \\ 161 & 131 & 81,37 \\ 159 & 123 & 77,36\end{array}$

$\begin{array}{ccc}0,094 & 21 & 53,85 \\ & 96 & 59,63 \\ & 106 & 66,67 \\ & 0 & 0,00\end{array}$

$\begin{array}{cccc}0,205 & 35 & 89,74 & 0,000 \\ & 151 & 93,79 & \\ & 156 & 98,11 & \\ & 0 & 0,00 & \end{array}$

Ainda não teve

CCU

\begin{tabular}{|c|c|c|c|c|c|c|c|c|c|c|}
\hline Sim & 20 & 14 & 70,00 & 0,537 & 11 & 55,00 & 0,674 & 20 & 100,00 & 0,598 \\
\hline Não & 340 & 267 & 78,53 & & 212 & 62,35 & & 322 & 94,71 & \\
\hline TOTAL & 360 & 281 & 78,06 & & 223 & 61,94 & & 342 & 95,00 & \\
\hline
\end{tabular}

*Salários mínimos/mês

A prática do exame demonstrou associação estatística significativa com a idade das mulheres e o início da atividade sexual. Ressalta-se que mulheres na faixa etária de 45 a 64 anos apresentaram maior percentual de prática adequada do que as mulheres mais jovens, assim como o conhecimento. Evidencia-se, ainda, que o início precoce da atividade sexual promove grande influência sobre a prática das mulheres, visto que quanto mais cedo foi o início da vida sexual menor foi a frequência de prática adequada. Em estudo realizado por Silveira e colaboradores (2016) foi demonstrado que a faixa etária reduzida teve relação significativa com o conhecimento e atitude inadequada. Além disso, no mesmo estudo notou-se que entre as adolescentes os três aspectos avaliados, conhecimento, atitude e prática, foram inadequados.

A escolaridade também apresentou diferença significativa, pois as mulheres com maior tempo de estudo apresentaram maior frequência de atitude adequada, perfil semelhante ao encontrado na Argentina por Gamarra e colaboradores (2005). Assim, maior grau de instrução entre as participantes do estudo demonstrou facilitar 
o acesso as informações e consequentemente permitir a mulher melhor compreensão sobre os benefícios do exame citopatológico.

Outro ponto importante encontrado no presente estudo foi a influência do número de filhos sobre a atitude das mulheres, dado que a baixa paridade refletiu em um maior percentual de atitude adequada em relação ao exame Papanicolaou. Resultado semelhante foi observado por Gamarra e colaboradores (2005) que também observou em seu estudo que mulheres que referiram não utilizar nenhum método contraceptivo apresentaram menor proporção de atitude adequada frente ao exame. Além disso, a multiparidade é descrita como um dos fatores de risco para a neoplasia cervical (MUÑOZ et al., 2006).

Em relação ao nível de conhecimento frente ao exame Papanicolaou, $98,89 \%$ das mulheres sabiam da existência do exame, sendo que $78,06 \%$ apresentaram conhecimento adequado. Logo, 20,83\% já tinham ouvido falar do exame, mas não indicaram a correta finalidade (Tabela 2). No que se refere à atitude, observou-se que $98,89 \%$ das mulheres consideravam a realização periódica do exame como necessária, porém, apenas $61,94 \%$ demonstraram atitude adequada, tendo como razão para a realização periódica do exame a prevenção do câncer de colo uterino (Tabela 2 e 3). A maioria das mulheres $(96,67 \%)$ já havia realizado o exame Papanicolaou alguma vez na vida, $39,72 \%$ realizavam o procedimento anualmente e 3,33\% nunca haviam feito. Constatou-se que $95,00 \%$ apresentaram prática adequada, tendo realizado o último exame há no máximo três anos (Tabela 2).

TABELA 2. Conhecimento, atitude e prática das mulheres em relação ao exame Papanicolaou. São Mateus, ES, Brasil, 2015. ( $N=360)$

\begin{tabular}{lcccc}
\hline & \multicolumn{2}{c}{ Adequado(a) } & \multicolumn{2}{c}{ Inadequado(a) } \\
& $\mathrm{N}$ & $\%$ & $\mathrm{~N}$ & $\%$ \\
\hline Conhecimento & 281 & 78,06 & 79 & 21,94 \\
Atitude & 223 & 61,94 & 137 & 38,06 \\
Prática & 342 & 95,00 & 18 & 5,00 \\
\hline
\end{tabular}

TABELA 3. Razões alegadas pelas mulheres para realização do exame Papanicolaou. São Mateus, ES, Brasil, 2015*.

\begin{tabular}{lcc}
\hline Razão & $\mathbf{N}$ & \% \\
\hline Orientação dos agentes comunitários de saúde & 76 & 21,11 \\
Orientação do médico & 85 & 23,61 \\
Prevenir câncer de colo uterino & 223 & 61,94 \\
Orientação de amigos ou parentes & 11 & 3,06 \\
Não sabe & 6 & 1,67 \\
\hline
\end{tabular}

${ }^{*}$ Foi possível mais de uma resposta para cada mulher (soma $>100 \%$ )

Observou-se, portanto, que o percentual de mulheres que relataram terem ouvido falar sobre o exame foi próximo ao encontrado por Andrade e colaboradores (2014) em Feira de Santana-BA $(99,1 \%)$, Neri e colaboradores (2013) em Picos-PI $(98,7 \%)$ e Albuquerque e colaboradores (2014) em Floriano-PI (94,5\%). Resultado muito diferente do estudo de Osis e colaboradores (2014) em que 69,5\% dos entrevistados nunca ouviram falar do HPV. Além disso, no presente estudo, a maioria das mulheres apresentaram conhecimento adequado, relatando que o exame Papanicolaou tem por objetivo a prevenção do câncer do colo uterino. Tal 
resultado foi superior ao demonstrado por outros autores, como Leite e colaboradores (2014) em Bauru-SP, em que 41,8\% das mulheres possuíam conhecimento adequado. Essa diferença poderia ser explicada pelas políticas educacionais desenvolvidas nas Unidades Básicas de Saúde do município, já que boa parte das entrevistadas havia participado de atividades educativas relacionadas ao tema e a participação dessas atividades na própria unidade de saúde foi significante nesse grupo de mulheres.

Nesse contexto, esperava-se que mulheres com conhecimento adequado possuíssem também atitude adequada, uma vez que ao saber sobre os riscos do câncer cervical e a importância de sua prevenção podem ser usados como motivos de realização do exame Papanicolaou. No entanto, identificou-se que apenas $64,94 \%$ apresentaram atitude adequada, contrapondo com o grau de conhecimento da amostra. Esse resultado pode estar relacionado ao fato de que as mulheres compreendem a importância do exame, mas o classificam apenas como método para identificar afecções ginecológicas em pacientes sintomáticas e não como instrumento de rastreamento de uma doença, comportamento esse já descrito na literatura (PINOTTI; CARVALHO; NISIDA, 1994 apud DAVIM et al., 2005).

As mulheres do grupo de risco devem realizar o exame regularmente de acordo com os resultados das análises anteriores. Se no intervalo de um ano, dois exames apresentarem-se normais, a espera para o próximo exame pode ser de três anos (INCA, 2016). Diante desse requisito, no estudo de Penaranda e colaboradores (2014) com mulheres hispânicas na fronteira dos Estados Unidos e o México, 62\% das entrevistadas demostraram adequação à prática do exame, contra $30,5 \%$ das mulheres argentinas (GAMARRA et al, 2005). Já no Nepal, Ranabhat e colaboradores (2014) não encontraram nenhum relato devido a alta porcentagem de mulheres que nunca fizeram o exame. Constata-se, portanto, que este parâmetro possui maior adequação entre as mulheres do norte do Espírito Santo (95,0\%).

Ainda que $96,67 \%$ das mulheres tenham realizado o teste de Papanicolaou alguma vez na vida, existem barreiras que dificultam a sua prática e o acesso ao serviço. Verificou-se que as principais dificuldades encontradas pelas mulheres para a realização do exame preventivo foram: vergonha $(36,39 \%)$, dificuldade para marcar consulta $(17,22 \%)$ e descuido ou esquecimento $(11,67 \%)$ (Tabela 4$)$.

TABELA 4. Barreiras encontradas pelas mulheres para a prática do exame Papanicolaou. São Mateus, ES, Brasil, 2015*.

\begin{tabular}{lcc}
\hline Barreira & $\mathbf{n}$ & $\mathbf{\%}$ \\
\hline Não solicitação do médico & 6 & 1,67 \\
Vergonha & 131 & 36,39 \\
Exame incomoda & 36 & 10,00 \\
Dificuldade para marcar consulta & 62 & 17,22 \\
Descuido ou esquecimento & 42 & 11,67 \\
Unidade Básica de Saúde distante & 5 & 1,39 \\
Falta de tempo & 25 & 6,94 \\
Outra & 62 & 17,22 \\
Nenhuma & 37 & 10,28 \\
\hline
\end{tabular}

${ }^{\star}$ Foi possível mais de uma resposta para cada mulher (soma $>100 \%$ )

Outro aspecto importante foram as barreiras relatadas pelas mulheres para a prática do exame citopatológico. A vergonha, por exemplo, foi considerada a dificuldade mais relevante que interfere na realização periódica do exame. Isso pode 
ser explicado pela imagem negativa que se constrói devido a exposição da genitália, principalmente quando o procedimento é realizado por profissionais do sexo masculino. Entende-se que a posição ginecológica promove mal estar e incômodo, sensações essas que poderiam ser minimizadas pelos profissionais de saúde a partir do desenvolvimento de uma relação de confiança e da manutenção de um ambiente agradável para mulher, informando-a sobre o procedimento e seus benefícios (CHUBACl; MERIGHI, 2005). Ressalta-se, ainda, que a dificuldade para marcação de consulta também atua como barreira para realização do exame preventivo, necessitando, portanto, da atenção dos gestores quanto à demanda atendida e à abrangência de cobertura de cada Unidade Básica de Saúde.

\section{CONCLUSÃO}

Os resultados desse estudo permitiram avaliar que a maioria das mulheres possuem conhecimento e prática adequados, identificando a correta finalidade do exame preventivo e o realizando periodicamente, conforme estabelecido pelo Ministério da Saúde. Diante dos fatos mencionados pode-se perceber um cenário promissor, uma vez que a realização do exame nos intervalos preconizados favorece o controle do câncer cervical, isto é, o alto percentual de prática adequada contribui com as ações de rastreamento da doença, reduzindo assim a mortalidade por essa neoplasia.

Em contrapartida, observou-se que boa parte da amostra apresentou atitude inadequada, constatando-se a necessidade das intervenções educativas que tenham por finalidade o incentivo à prevenção do câncer do colo do útero, garantindo a conscientização sobre a importância do exame Papanicolaou.

As principais barreiras citadas para a realização do exame, a vergonha e o descuido com a saúde, podem estar influenciando a atitude das mulheres em relação ao procedimento. Esse cenário exige o aperfeiçoamento do método de trabalho de modo a melhorar a compreensão das mulheres acerca dos benefícios do exame. Para isto, é imprescindível que as ações de saúde da mulher busquem o atendimento humanizado a fim de criar uma relação de confiança entre a paciente e o profissional de saúde.

\section{REFERÊNCIAS}

ALBUQUERQUE, C. L. F.; COSTA, M. P.; NUNES, F.M.; FREITAS, R. W. J. F.; AZEVEDO, P. R. M.; REGO, J. V.; BARRETO, H. M. Knowledge, attitudes and practices regarding the Pap test among women in northeastern Brazil. São Paulo Medical Journal, São Paulo, v.132, n.1, p.3-9, 2014. Disponível em: <http://dx.doi.org/10.1590/1516-3180.2014.1321551>. doi: 10.1590/15163180.2014.1321551

ANDRADE, M. S.; ALMEIDA, M. M. G. de; ARAUJO, TM. De; SANTOS, K. O. B. Fatores associados a não adesão ao Papanicolau entre mulheres atendidas pela Estratégia Saúde da Família em Feira de Santana, Bahia, 2010. Epidemiologia e Serviços de Saúde, Brasília, v.23, n.1, p.111-120, 2014. Disponível em: <http://dx.doi.org/10.5123/S1679-49742014000100011>. doi: 10.5123/S167949742014000100011

ARBYN, M.; RAIFUA, A.O.; WEIDERPASS, E.; BRAY, F.; ANTTILA, A. Trends of cervical cancer mortality in the member states of the European Union. European 
Journal of Cancer, v.45, n.15, p.2640-2648, 2009a. Disponível em: <http://dx.doi.org/10.1016/j.ejca.2009.07.018>. doi: 10.1016/j.ejca.2009.07.018

BRASIL. Ministério da Saúde. Secretaria de Atenção à Saúde. Departamento de Atenção Básica. Controle dos cânceres do colo do útero e da mama. Editora do Ministério da Saúde. 2. ed. Brasília, 2013. Disponível em: http://bvsms.saude.gov.br/bvs/publicacoes/controle_canceres_colo_utero_2013.pdf

BRENNA, S. M. F.; HARDY, E.; ZEFERINO, L. C.; NAMURA, I. Conhecimento, atitude e prática do exame de Papanicolaou em mulheres com câncer de colo do uterino. Cadernos de Saúde Pública, p. 909-914, 2001. Disponível em: <http://dx.doi.org/10.1590/S0102-311X2001000400024>. doi: 10.1590/S0102311X2001000400024

CHUBACI, R. Y. S.; MERIGHI, M. A. B. Exame para detecção precoce do câncer cérvico-uterino: vivência de mulheres das cidades de Kobe e Kawasaki, Japão e São Paulo, Brasil. Revista Brasileira de Saúde Materno Infantil, Recife, v. 5, n. 4, p. 471-481, dez. 2005. Disponível em: <http://dx.doi.org/10.1590/S151938292005000400011>. doi: 10.1590/S1519-38292005000400011

DAVIM, R. M. B.; TORRES, G. de V.; SILVA, R. A. R da; SILVA, D. A. R. da. Conhecimento de mulheres de uma Unidade Básica de Saúde da cidade de Natal/RN sobre o exame de Papanicolau. Revista Escola Enfermagem USP, v. 39, n. 3, p. 296-302, 2005. Disponível em: <http://dx.doi.org/10.1590/S008062342005000300007>. doi: 10.1590/S0080-62342005000300007

GAMARRA, C. J.; PAZ, E. P. A.; GRIEP, R. H. Conhecimentos, atitudes e prática do exame de Papanicolaou entre mulheres argentinas. Revista de Saúde Pública, São Paulo, v. 39, n. 2, p. 270-276, abr. 2005. Disponível em: <http://dx.doi.org/10.1590/S0034-89102005000200019>. doi: 10.1590/S003489102005000200019

IARC. International Agency for Research on Cancer. World Health Organization. Estimated Cancer Incidence, Mortality and Prevalence World Wide in 2012. Globocan 2012. Disponível em: <http://globocan.iarc.fr/Pages/fact_sheets_ cancer.aspx> Acesso em: 12 dez. 2017.

IBGE. Instituto Brasileiro de Geografia e Estatística. Censo demográfico, 2010. Disponível em: https://cidades.ibge.gov.br/brasil/es/sao-mateus/panorama. Acesso em: 12 dez. 2017.

INCA. Instituto Nacional de Câncer José Alencar Gomes da Silva. Diretrizes Brasileiras para o Rastreamento do Câncer do Colo do Útero. 2. ed. Rio de Janeiro, 2016. Disponível em: http://www.citologiaclinica.org.br/site/pdf/documentos/diretrizes-para-o-rastreamentodo-cancer-do-colo-do-utero_2016.pdf

INCA. Instituto Nacional de Câncer José Alencar Gomes da Silva. HPV e Câncer. Ministério da Saúde. Rio de Janeiro. Disponível 
em: <http://www2.inca.gov.br/wps/wcm/connect/tiposdecancer/site/home/colo_utero/ hpv-cancer-perguntas-mais-frequentes> Acesso em: 12 dez. 2017c

INCA. Instituto Nacional de Câncer José Alencar Gomes da Silva. Ministério da Saúde. Estimativas para o ano de 2016 das taxas brutas de incidência por 100 mil habitantes e do número de casos novos de câncer, segundo sexo e localização primária. Brasil (Consolidado). Estimativa 2016: Incidência de Câncer no Brasil. Rio de Janeiro. Disponível em: <http://www.inca.gov.br/estimativa/2016/tabelaestados.asp?UF=BR> Acesso em 12 dez. 2017a.

INCA. Instituto Nacional de Câncer José Alencar Gomes da Silva. Ministério da Saúde. Controle do Câncer do Colo do Útero: Detecção Precoce. Rio de Janeiro. Disponível em: <http://www2.inca.gov.br/wps/wcm/connect/acoes_programas/site/home/nobrasil/pro grama_nacional_controle_cancer_colo_utero/deteccao_precoce> Acesso em: 12 dez. $2017 \mathrm{~b}$.

LEITE, M. F.; VITTA, F. C. F. de; CARNAZ, L.; CONTI, M. H. S. de; MARTA, S. N.; et al. Conhecimentos e prática das mulheres sobre câncer de colo do útero de uma unidade básica de saúde. Revista Brasileira de Crescimento e Desenvolvimento Humano, São Paulo, v. 24, n. 2, p. 208-213, 2014. Disponível em: http://pepsic.bvsalud.org/pdf/rbcdh/v24n2/pt_14.pdf

MUÑOZ, N.; CASTELLSAGUÉ, X.; DE GONZÁLEZ, A. B.; GISSMANN, L. HPV in the etiology of human cancer. Vaccine, v. 24, ago. 2006. Disponível em: < http://dx.doi.org/10.1016/j.vaccine.2006.05.115>. doi: 10.1016/j.vaccine.2006.05.115

NERI, É. de A. R.; MOURA, M. S. S. de; PENHA, J. C. da; REIS, T. G. O. dos; AQUINO, P. de S.; PINHEIRO, A. K. B. Conhecimento, atitude e prática sobre o exame papanicolaou de prostitutas. Texto \& Contexto - Enfermagem, Florianópolis, v. 22, n. 3, p. 731-738, set. 2013. Disponível em: <http://dx.doi.org/10.1590/S0104-07072013000300020> doi: 10.1590/S010407072013000300020

OPAS. Organização Pan-Americana da Saúde. Nota de orientação da OPAS/OMS. Prevenção e controle de amplo alcance do câncer do colo do útero: um futuro mais saudável para meninas e mulheres. Washington, DC: OPAS, 2013.

OSIS, M.J.D.; DUARTE, G.A.; SOUSA, MH. Conhecimento e atitude de usuários do SUS sobre o HPV e as vacinas disponíveis no Brasil. Revista de Saúde Pública, v. 48, n. 1, p. 123-133, 2014. Disponível em: http://dx.doi.org/10.1590/S00348910.2014048005026. DOI: 10.1590/S0034-8910.2014048005026

PENARANDA E.; MOLOKWU J.; HERNANDEZ I.; SALAIZ R.; NGUYEN N.; BYRD T.; SHOKAR N. Attitudes toward self-sampling for cervical cancer screening among primary care attendees living on the US-Mexico border. Southern Medical Journal, v. 107, ed. 7, p. 426-432, 2014. Disponível em: <http://dx.doi.org/10.14423/SMJ.0000000000000132>. doi: 10.14423/SMJ.0000000000000132. 
PINOTTI, J. A.; CARVALHO, J. P.; NISIDA, A. C. T. Implantação de programa de controle do câncer de colo uterino. Revista Brasileira de Ginecologia e Obstetrícia, v. 5, n. 1, p. 5-11, 1994.

RANABHAT S.; TIWARI M.; DHUNGANA G.; SHRESTHA R. Association of knowledge, attitude and demographic variables with cervical Pap smear practice in Nepal. Asian Pacific Journal of Cancer Prevention, v. 15, ed. 2; p. 8905-8910, 2014. Disponível em: <http://dx.doi.org/10.7314/APJCP.2014.15.20.8905>. doi: 10.7314/APJCP.2014.15.20.8905.

SILVEIRA, N. S. P.; VASCONCELOS, C. T. M.; NICOLAU, A. I. O.; ORIÁ, M. O. B.; PINHEIRO, P. N. C.; PINHEIRO, A. K. B. Conhecimento, atitude e prática sobre o exame colpocitológico e sua relação com a idade feminina. Revista Latino Americana de Enfermagem, v. 24, p. 01-07, 2016. Disponível em: https://doi.org/10.1590/1518-8345.0700.2699. doi: 10.1590/1518-8345.0700.2699 